\title{
Resonator Sensors for Rheological Properties - Theory and Devices
}

\author{
Erwin K. Reichel ${ }^{*}$, Martin Heinisch, Bernhard Jakoby \\ Institute for Microelectronics and Microsensors, Johannes Kepler University Linz, Austria \\ *) erwin.reichel@jku.at
}

\begin{abstract}
Resonators are sensitive to their external loading. When a surface is exposed to a liquid, rheological properties can be measured. In contrast to laboratory methods, resonator sensors feature high sensitivity at low viscosity and applicability for online monitoring tasks. On the other hand, the accuracy decreases significantly at higher viscosities. Here, the concept and theory is investigated with the example of an electrodynamic resonator sensor where a sessile droplet of sample liquid is deposited on the sensitive area. The in-plane motion leads to a dominant shear-wave interaction between the fluid and the oscillating structure.
\end{abstract}

Key words: viscosity sensor, resonator, shear-wave

\section{Introduction}

Viscosity is an important property for the processing and application of liquids. A variety of laboratory viscometric methods are available, often requiring either delicate instrumentation, skilled workers, or both. Some process line sensors are available but restricted to simple (Newtonian) liquids and limited in viscosity range. Solvents and pure, low viscous oils are examples for Newtonian liquids, where a single viscosity parameter is sufficient for the whole range of applicable shear rates and flow conditions. Suspensions like paint, emulsions, surfactant and lipid solutions are examples for fluids exhibiting non-Newtonian behavior. For these materials, a wide spectrum of rheological properties can be measured. A first extension is considering a shear-rate dependent viscosity $\eta(\dot{\gamma})$, known as the flow curve [1]. Increasing viscosity with shear-rate is known as shearthickening, the opposite as shear-thinning both phenomena are observed in concentrated colloidal suspensions. Another approach is the application of an oscillatory deformation. Where simple liquids show a reaction force that is $\frac{\pi}{2}$ out of phase to the displacement, an elastic solid leads to an in-phase reaction force. Everything in between falls in the category of (linear) viscoelasticity. The probed property is usually the complex shear modulus as a function of frequency $G(\mathrm{i} \omega)$ (in rheology literature often denoted as $G^{*}$ ).

The use of mechanical resonators to characterize fluids is becoming an interesting alternative to conventional methods. Distinct features are a high sensitivity at low viscosities, the possibility of miniaturization, and onlinemeasuring capability. The theory of fluidstructure interaction for these devices is certainly more involved, and the flow field is no longer viscometric in the strict sense, because it depends on the fluid properties. These issues are mainly avoided when the oscillation is dominantly in-plane, leading to shear-wave interaction. Current challenges of this method are extending the viscosity range and probing viscoelastic properties in a wide frequency range.

\section{Theory \\ Rheology Basics}

The flow curve $\eta(\dot{\gamma})$ describes the dependency of viscosity on the shear-rate. It is usually measured under conditions of uniform (shear) deformation throughout the sample volume, as realized, or closely approximated, by Couette or cone-plate geometries. Some fluids need to overcome a certain stress threshold, known as the yield stress, to make a transition from elastic to viscous behavior. Common examples of yield-stress fluids are mayonnaise, toothpaste, and various gels. These materials do not really behave like liquids as they can easily maintain a deformed surface and it requires an extra effort to make them flow. But even obvious liquids can alter their flow properties when increasing the rate of deformation. For polymer melts the most 
common behavior is shear-thinning: Below a certain shear-rate the viscosity is strainindependent but decreases at higher strain rates. An interesting relation has been found emperically in the form of the Cox-Merz rule [2]. This relation states that the (non-linear) flowcurve can be obtained from linear oscillatory measurements by substituting the angular frequency $\omega$ of the magnitude of complex viscosity $\eta(\mathrm{i} \omega)=\mathrm{G}(\mathrm{i} \omega) / \mathrm{i} \omega$ by the shear rate $\dot{\gamma}$ : $\eta(\dot{\gamma})=|\eta(\mathrm{i} \omega)|_{\omega=\dot{\gamma}}$. This rule holds for linear polymers and blends thereof, but generally not for more structured media like suspensions and emulsions.

The complex shear modulus contains valuable information by itself. The limiting behavior towards zero frequency reveals if the material appears as solid or liquid, as the elastic contribution vanishes for the latter. The high frequency domain is important to reveal the highest relaxation time constant of dissipative processes. In between, the rheological spectrum is used to quantify different mechanisms of relaxation and dissipation. With conventional rheometers, the frequency range of observation is limited to around $100 \mathrm{~Hz}$. At higher frequencies, either the eigenfrequency of the instrument is approached, or the uniform shear profile changes to a wave-like regime. Understanding these high-frequency effects inspires resonator-based methods, which in contrast to conventional methods feature high sensitivity at low viscosities and the potential for solid-state online sensors.

\section{Resonator Modeling}

Here we will examine a resonator sensor as a system of a transfer function associated with a frequency response that is sensitive to the quantity to be measured. The sensitive element is a mechanical structure that can be set into vibration, exhibiting (at least one) resonance frequency. Certain parts of this structure are exposed to a fluid. The vibration generates an oscillatory flow, which acts back on the structure, hence altering its frequency response. All amplitudes are small enough so that the overall system behavior is linear. The latter requirement has to be checked for each individual case. The term associated with convection in the equations of fluid motion has to be negligibly small and geometrical or material non-linearities in the resonator have to be excluded.

Commonly, the mechanical transfer function is described in terms of velocity $\boldsymbol{v}$ resulting from force $\boldsymbol{F}$. The equation of motion for a distributed system can be described by a complex velocity at one representative point and an associated mode-shape by means of eigenmode decomposition [3]. For most practical cases the motion can be decomposed into a potentially infinite series of (lumped) second order oscillators, where only one or a few modes are relevant. The system possesses a transduction mechanism which allows the electrical measurement of the mechanical transfer function, e.g. by piezoelectric, piezoresistive, or electrodynamic effects.

\section{Fluid interaction}

Accurate models for the fluid interaction are generally hard to find. For simple geometries analytical or efficient numerical models can be implemented. The shear-wave interaction of a plate oscillating in its own plane is an example of the first kind. The flow around an infinitely thin blade is exemplarily for the second. The flow reaction force has the property that it scales with the (complex) surface velocity and exhibits dependence on frequency, fluid density, and complex shear modulus.

\section{Devices}

\section{Plate Resonator}

Shear-waves are preferred for viscometric and rheometric methods. The flow field is still dependent on the fluid properties, but in a defined way where an analytical solution for the reaction force is available. For this solution to be applicable, the device dimensions have to be large compared to the shear-wave penetration depth. This poses certain limitations on the miniaturization of such devices. Investigations on silicon-micromachined plate resonators have shown, that the fluidinteraction cannot be assumed to be of the shear-wave type only, but quality factors are still good for viscometric purposes [4].

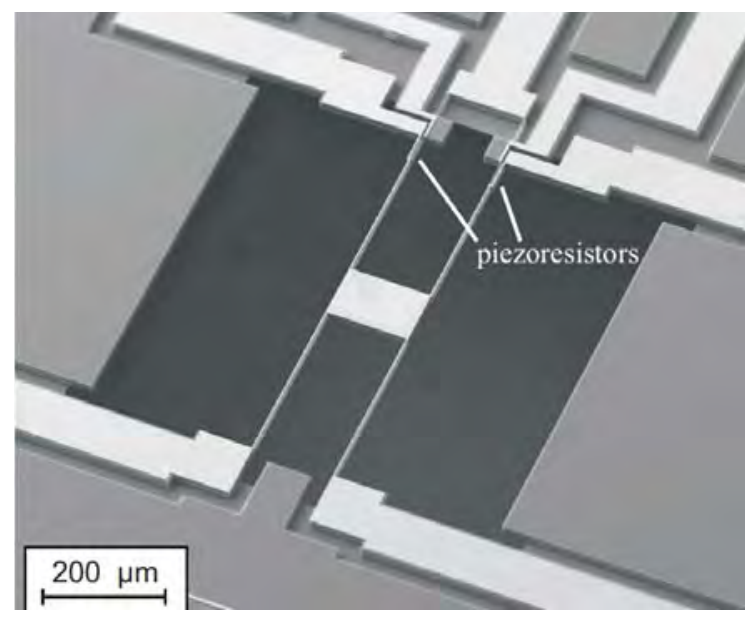

Fig. 1. SEM micrograph of a micromachined plate resonator viscosity sensor with Lorentz force excitation and piezoresistive readout (from [4]). 


\section{Micromachined Devices}

In recent years, a variety of micromachined resonator devices were introduced. Cantilevers are widely used in atomic force microscopy (AFM), in vacuum, gas, and liquid environment. Fluid-structure interaction in the latter case requires certain attention, but is merely treated as a spurious effect for the analysis of surface topology or biochemical texture. Clampedclamped beams are used for Lorentz-force excitated miniature viscometers, but pre-stress effects hamper stable sensor operation.

A micromachined device in silicon technology was presented by Riesch et al [4], where a rectangular plate is driven by Lorentz-forces and read out by integrated piezoresistors, see Fig. 1. A slight disadvantage is the high area moving out-of-plane compared to the in-plane surfaces of the top and bottom of the suspended plate.

The miniaturization of fluid sensors is a technological achievement and will most likely find its way into microfluidic analysis systems. On the other hand, for widespread application in the laboratory or in the field, these devices are often too sensible against contamination and rough environment. A spring-plate resonator built from a single metal sheet, based on earlier work [5], is shown in Fig. 2.

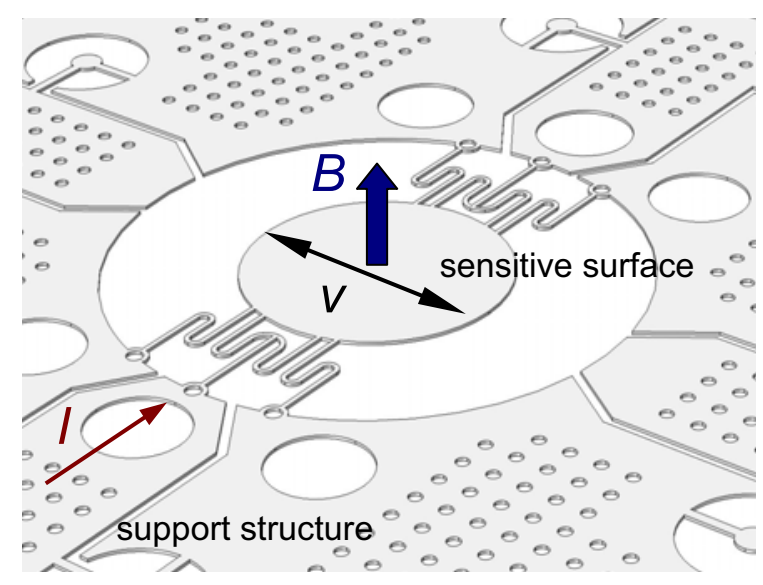

Fig. 2. Schematic of the metallic plate resonator excited by the Lorentz force generated by the sinusoidal current $\boldsymbol{I}$ and the static magnetic field $\boldsymbol{B}$. The diameter of the round sensitive surface is $8 \mathrm{~mm}$. The support structure is fixed to a PCB, the springs and plate are free standing, and the sample liquid is placed on the circular area.

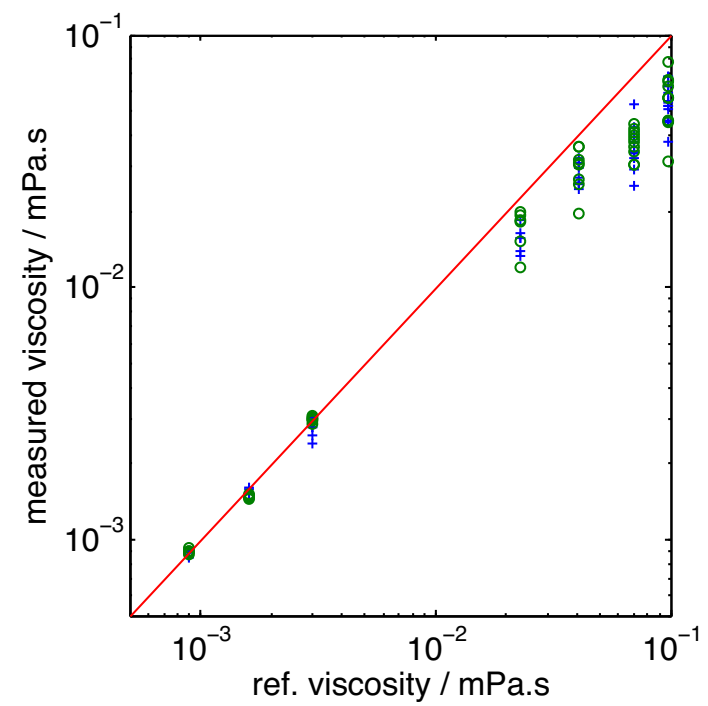

Fig. 3. Viscosity as measured by the resonator against the reference viscosity, measured by a coneplate rheometer. The blue (+) and green (o) symbols correspond to values derived from damping and resonance frequency, respectively - deviation from the red line at higher viscosity is explained by nonperfect shear-wave interaction.

\section{Metallic Spring-Plate Resonator}

The device consists of a lithographically structured metal sheet (nickel-brass) fixed on a PCB. The sensitive element is a plate suspended on spring structures. A permanent magnetic field is aligned perpendicular to the plate and a sinusoidal current is applied so that Lorentz forces cause an in-plane motion. The geometry of the spring structure and effective mass define a sharp resonance. Close to the resonance frequency, a sufficiently high voltage is induced which can be measured over two terminals. The external electronics allow a rapid measurement of the frequency response. For this purpose, an off-the-shelf audio-interface is used. The recorded data set shows a spurious baseline (caused by ohmic resistance and signal transformer characteristics) and the electromechanical resonance peak [6]. The frequency response is measured within five seconds time.

\section{Measurements}

Although fully immersing the resonator in the sample liquid is possible, it is sufficient to deposit a droplet of defined volume (100 microliters) on the sensitive surface. The electrical transfer function incorporating a spurious coupling $K_{0}$ is modeled by a secondorder oscillator: 
$H(\mathrm{i} \omega)=K\left[\frac{\omega_{\mathrm{r}}}{Q}+\mathrm{i}\left(\omega-\frac{\omega_{\mathrm{r}}^{2}}{\omega}\right)\right]^{-1}+K_{0}$

with (angular) resonance frequency $\omega_{\mathrm{r}}$, quality factor $Q$, and electromechanical coupling factor $K$. The latter depends on device geometry and the magnetic field $B$. The quantity to be measured is the complex fluid loading, characterized by a change in resonance frequency and additional damping. The analytical solution for a pure shear-wave interaction force is

$F_{\mathrm{fl}}=A \sqrt{G(\mathrm{i} \omega \rho)}$,

where $A$ is the area of interaction, $G$ the frequency dependent shear modulus, and $\rho$ the density. In order to calculate the fluid loading from the measured resonance parameters, four calibration constants, $C_{1}, C_{2}, C_{3 a}$, and $C_{3 \mathrm{~b}}$, are required:

$$
\begin{aligned}
& \Re\{\sqrt{G \rho}\}=\left(\frac{1}{Q}-C_{1}\right) \frac{1}{\omega_{\mathrm{r}} C_{3 a}} \\
& \Im\{\sqrt{G \rho}\}=\left(\frac{1}{\omega_{\mathrm{r}}^{2}}-C_{2}\right) \frac{\omega_{\mathrm{r}}}{C_{3 b}} .
\end{aligned}
$$

The constants $C_{3 a}$, and $C_{3 b}$ would be equal for a pure shear-wave interaction as described by Eq. 2. The real part, Eq. 3, is derived from the Q-factor, and the imaginary part, Eq. 4, from the change in resonance frequency. Combining these, the complex shear modulus at the resonance frequency can be calculated, knowing the density. In the case of a Newtonian liquid, the shear modulus is $G(\mathrm{i} \omega)=\mathrm{i} \omega \eta$, and the viscosity can be derived from either Eq. 3 or 4.

Table 1 shows a series of test liquids prepared from Glycerol and deionized water. The reference values for viscosity were measured using a Brookfield DVIII cone-plate rheometer, density values were taken from literature.

Tab. 1: Aqueous Glycerol mixtures

\begin{tabular}{|c|c|c|c|}
\hline Sample & $\begin{array}{c}\text { Glycerol } \\
\text { content } \\
\%\end{array}$ & $\begin{array}{c}\text { Viscosity } \\
\text { mPa.s }\end{array}$ & $\begin{array}{c}\text { Density } \\
\mathrm{kg} \cdot \mathrm{m}^{-3}\end{array}$ \\
\hline $\mathrm{G} 0$ & 86.8 & $97 \pm 3$ & 1223 \\
\hline $\mathrm{G} 1$ & 84.7 & $70 \pm 1$ & 1214 \\
\hline $\mathrm{G} 2$ & 79 & $40.8 \pm 1$ & 1201 \\
\hline $\mathrm{G} 3$ & 73.1 & $22.9 \pm 0.3$ & 1178 \\
\hline $\mathrm{G} 4$ & 39 & $3.0 \pm 0.1$ & 1089 \\
\hline $\mathrm{G} 5$ & 21.5 & $1.6 \pm 0.1$ & 1044 \\
\hline $\mathrm{H}_{2} \mathrm{O}$ & 0 & $0.9 \pm 0.1$ & 1000 \\
\hline
\end{tabular}

In Fig. 3, the result of the sensor performance is shown. Good sensitivity and agreement is observed for low viscous fluids. At viscosity values higher than about $20 \mathrm{mPa} . \mathrm{s}$, a deviation from the plane shear-wave behavior (straight line) is observed. Additionally, the statistical error increases dramatically, so this value is assumed to constitute the measurement range of the current method.

\section{Conclusions and Perspectives}

The presented plate resonators are promising devices for sensors measuring the complex shear modulus because of their robust construction, integrated readout, and welldefined fluid-structure interaction. A current challenge is the extension of viscosity range to higher values, as most non-Newtonian liquids exhibit high base viscosity values. Nevertheless, potential applications can be found in viscosity-sensitive concentration measurement, the characterization of dilute to semi-dilute suspensions, and fast measurement of low sample volume.

\section{Acknowledgements}

We are indebted to the Austrian Centre of Competence in Mechatronics (ACCM) for financial support.

\section{References}

[1] C. W. Macosko, Rheology: Principles, Measurements, and Applications. Wiley- $\mathrm{VCH}$, 1994

[2] W.-M. Kulicke and R.S. Porter, Relation between steady shear flow and dynamic rheology, Rheol. Acta 19, 601-605, 1980

[3] E.K. Reichel, B. Jakoby, Microsensors Based on Mechanically Vibrating Structures, Advanced Dynamics and Model-Based Control of Structures and Machines, 2012, pp 183-193

[4] C. Riesch, E.K. Reichel, A. Jachimowicz, J. Schalko, P. Hudek, B. Jakoby and F. Keplinger, A suspended plate viscosity sensor featuring inplane vibration and piezoresistive readout, $\mathrm{J}$. Micromech. Microeng. 19 075010, 2009, doi:10.1088/0960-1317/19/7/075010

[5] E.K. Reichel, C. Riesch, F. Keplinger, C.E.A. Kirschhock, B. Jakoby, Analysis and experimental verification of a metallic suspended plate resonator for viscosity sensing, 162/2, 2010, 418-424

[6] E. K. Reichel, M. Heinisch, B. Jakoby, J. Vermant, C.E.A. Kirschhock, Viscoelasticity sensor with resonance tuning and low-cost interface, Proc. Eurosensors XXV, September 47, 2011 